\title{
ОТ ЛОГИКИ ЭПИСТЕМОЛОГИИ К ЭПИСТЕМОЛОГИИ ЛОГИКИ
}

\begin{abstract}
В.В. Горбатов
Эпистемическая логика появилась в конце 1950-х - начале 1960-х годов благодаря блестящим работам К. Геделя, Г. фон Вригта, А. Прайора и, прежде всего, Я. Хинтикки, обнаруживших, что структуры и закономерности, связанные с понятиями «знание», «мнение», «убеждение», могут быть представлены в виде аксиоматических дедуктивных систем. Она развивалась бок о бок с модальной и интенсиональной логиками, долгое время разделяя с ними довольно удобный и философски привлекательный способ интерпретации - семантику возможных миров.

Но оказалось, что столь близкие изначально области исследования ожидала разная судьба. По сравнению с тем, насколько важную роль играет модальная логика для современных метафизиков (С.Крипке, Д. Льюис, А. Плантинга, Р. Чизолм), маргинальное положение эпистемической логики в философии познания и науки просто поразительно. Даже ее наиболее выдающиеся и многообещающие технические результаты воспринимаются философами весьма прохладно и остаются в стороне от эпистемологического мэйнстрима. Чем же объясняется такая парадоксальная ситуация?
\end{abstract}

\section{1 Дихотомия «контекстов открытия» и «контекс- тов обоснования»}

Прежде всего, в этом следует винить характерное для позитивизма и идущее еще от Г. Рейхенбаха противопоставление «контекстов откры-

Актуальні проблеми духовності:

зб. наук. праць / Ред.: Я.В. Шрамко

Вип. 10. - Кривий Ріг, 2009, 219-233 
тия» и «контекстов обоснования». Сегодня большинство философов науки признают, что логическому анализу доступны лишь последние; что же касается первых, то они, как правило, связаны с неформализуемыми и неэксплицируемыми аспектами познания. По словам Карла Поппера, «вопрос о путях, по которым новая идея - будь то музыкальная тема, драматургический конфликт или научная теория - приходит человеку, может представлять существенный интерес для эмпирической психологии, но он совершенно не относится к логическому анализу научного знания» $[1$, с. 26].

А дальше обычно используется аргумент, напоминающий знаменитую дилемму халифа Омара: «Если ваши книги согласны с Кораном, то они излишни; если они не согласны с Кораном, то они вредны, в любом случае их надо сжечь». Действительно, если систематические регулярности, связанные с нашим использованием эпистемических понятий, имеют место только в «контекстах обоснования», то для их анализа не требуется никакая специальная эпистемическая логика, поскольку обоснованиями и доказательствами занимается как раз логика обычная. Если же мы пытаемся исследовать в формально-аналитических терминах «контексты открытия», то тем самым заведомо искажаем их смысл, связанный со свободным, иногда даже стихийным творческим поиском. В любом случае, в рамках такой парадигмы статус эпистемической логики оказывается под большим вопросом.

\section{2 Слабая эффективность «сильной» рационально- сти}

Вторая причина, по которой эпистемическая логика оказалась не слишком популярна среди философов,- неисправимо сильные идеализации, на которых она изначально строилась. Дело в том, что невозможно сформулировать ни одну достаточно богатую и интересную в философском плане систему эпистемической логики, не приписав познающему субъекту минимальные рациональные способности. Но оказывается, что в рамках нормальных эпистемических логик с крипкевской семантикой даже «минимальная» рациональность, необходимая для философской оправданности $K$-оператора (оператора «известно, что»), является чересчур сильной. В частности, мы должны принять

(i) аксиому дедуктивной связности $K_{a}(p \rightarrow q) \rightarrow\left(K_{a} p \rightarrow K_{a} q\right)$ (если $a$ знает, что из $p$ следует $q$, то из известности для $a$ факта $p$ следует известность для $a$ факта $q$ ), а также 
(ii) аксиому истинности $K_{a} p \rightarrow p$ (если известно, что $p$, то $p$ истин$\mathrm{HO})$.

В большинстве стандартных систем эпистемической логики принимаются также аксиомы

(iii) позитивной интроспекции $K_{a} p \rightarrow K_{a} K_{a} p$ (если $a$ знает, что $p$, то $a$ знает, что $a$ знает, что $p$ ) и

(iv) негативной интроспекции $\sim K_{a} p \rightarrow K_{a} \sim K_{a} p$ (если $a$ не знает, что $p$, то $a$ знает, что $a$ не знает, что $p$ ).

Что значит для познающего субъекта $a$ знать высказывание $p$ ? Это значит, что во всех мирах, совместимых с тем, что известно $a$ (т. е. $a$-достижимых), высказывание $p$ должно быть истинным. Принятие аксиом (i-iv) приводит к тому, что отношение достижимости между возможными мирами, ассоциированное с индивидом $a$, должно быть рефлексивным, симметричным и транзитивным, то есть представлять собой отношение эквивалентности. Не секрет, что системы именно с таким отношением достижимости оказываются подходящими для наибольшего числа приложений.

В порыве эпистемического аскетизма, можно, конечно, отказаться от аксиом (ii-iv) или ослабить их подходящим образом. Но разве вправе мы подвергать сомнению аксиому (i), выражающую «святая святых» эпистемической логики - способность индивида получать знание путем дедукции? Оказывается, и это вполне реально. Еще в статье 1972 г. «Возможна ли эпистемическая логика?» [9] Макс Хокатт сформулировал проблему «логического всеведения»: если индивид знает некое высказывание, то обязан ли он знать все логические следствия этого высказывания? Отвечая на данный вопрос отрицательно, Хокатт приходит к выводу: нет никаких гарантий, что индивид распознает некое суждение, которое мы ему приписываем, как логически эквивалентное тому, с которым он действительно согласен.

Насколько же идеализированный субъект познания, обладающий рациональными характеристиками в смысле аксиом (i-iv), совпадает с реальным субъектом познания? Насколько адекватно «возможные миры» и заданные на их множестве отношения достижимости отражают формальную онтологию познавательного процесса?

Очевидно, у нас есть веские основания констатировать, что практическая применимость описанных выше логических конструкций в 
сколь-нибудь реалистичном исследовании человеческого познания весьма сомнительна. Дело в том, что лежащие в основе эпистемической логики первого поколения теоретические идеализации базируются на понятии «сильной рациональности», главные слагаемые которого, на наш взгляд, таковы:

1) Пассивность познающего субъекта, исключение его из сферы действия логических операторов. Технически это выражается в том, что в стандартной эпистемической логике для субъекта зарезервирована лишь одна роль - в лучшем случае он служит индексом для $K$ оператора. В семантическом плане это значит, что субъект лишь индицирует определенные алгебраические ограничения, накладываемые на отношение достижимости между мирами (сценариями). Другими словами, он оказывается вне области действия K оператора (да и всех других логических символов), представляя собой некий метасистемный параметр. Это не тот субъект, который получает данные, меняет свое мнение, взаимодействует с другими субъектами и имеет с ними общие знания, а скорее некая мертвая проекция заранее приписанных эпистемическому оператору формальных свойств.

2) Статичность познавательных конструкций, игнорирование механизмов фиксации, развития и пересмотра знаний, функционирующих в реальном человеческом мышлении. Фактически получается, что если субъект единожды признал некое высказывание в качестве истинного, то никакая дополнительная информация уже не заставит его отказаться от своего знания. А если заставит, значит он не обладал знанием в строгом смысле слова. Вряд ли стоит напоминать, что даже в строгих науках, не говоря уже об обыденном познании, мы не встречаем примеров того, как это требование может быть выполнено в полном объеме.

3) Полнота и непротиворечивость «миров знания». Представление о том, что когда бы ни осуществлялись познавательные процессы, они протекают в условиях полной и непротиворечивой информации, что знание индивида соотносится не с отдельными частями, фрагментами мира, но со всем миром в целом. Такой холизм зачастую превращается, по меткому выражению Я. Хинтикки, в «интеллектуальную мегаломанию», заставляющую нас мыслить эпистемически возможные миры как некие полноценные вселенные, существующие независимо от нашего мира и несводимые к нему. На самом же деле практика познания подсказывает, что субъект всегда имеет дело с ситуациями, т. е. с ограниченными частями мира, и реальность этих альтернативных ситуаций (совместимых либо несовместимых со знаниями субъекта) 
носит вовсе не метафизический, а прагматический характер.

Необходимо отметить, что по историческому совпадению ранняя эпистемическая логика, опиравшаяся на описанную выше модель рациональности, находилась в стороне от тенденций, наметившихся в сфере гуманитарных наук. Уже в конце 1950-х гг. в экономике, которая к тому времени довольно успешно оперировала понятиями «рационального выбора» и «компетентного принятия решений», появилось представление об «ограниченной», или «частичной рациональности» (впервые этот термин был использован Г. Саймоном в 1957 г.). Очень скоро данное представление стало популярным в кибернетике, AI-исследованиях, нелинейном программировании, лингвистике, когнитивной психологии и даже в биологии. Вероятно, это послужило одной из причин невнимательного отношения к эпистемической логике со стороны специальных дисциплин, а впоследствии привело и к тому, что наиболее острые, актуальные философские вопросы о рациональности чаще ставились не в абстрактно-логических терминах, а в терминах этих частных наук.

\section{3 Универсалистская парадигма в логике}

Наконец, третьей причиной маргинализации эпистемической логики в философском дискурсе стали, на наш взгляд, глубинные особенности той классической парадигмы, которая направляла развитие символической логики вообще со времен Фреге и Рассела. Если воспользоваться терминологией Ван Хейеноорта [4], в ХХ веке традиция «логика как язык» возобладала над традицией «логика как исчисление». Первая - традиция Фреге, - по его мнению, основана на трактовке логического символизма как универсального языка (lingua characteristica), следствием чего является невозможность сказать что-либо вне рамок самой системы, т. е. все метасистемные вопросы оказываются заведомо исключенными из рассмотрения. Вторая же традиция, представленная именами Пирса, Буля, Шредера, напротив, рассматривает логический символизм как формальное исчисление (calculus ratiocinator), допускающее различные интерпретации в разных областях дискурса.

Важно понимать, что универсалистская парадигма не просто предполагает единый, нестратифицированный универсум рассмотрения (вот она, главная причина «интеллектуальной мегаломании»), но и рассматривает язык как неустранимый посредник любой концептуализации такого универсума. Согласно этой точке зрения, мы не мо- 
жем отделить себя от своих понятий, поскольку у нас нет возможности остановить нашу концептуальную практику без их утраты. Язык (говоря словами Витгенштейна, «единственный язык, который я понимаю») - есть неустранимый посредник между мною и миром, посредник, без которого я не могу обойтись. Таким образом, я не могу выйти за пределы своего языка (и воплощаемой им понятийной системы) и видеть его со стороны (см.: [2]).

Каким же образом универсалистская парадигма могла помешать полноценному развитию и философскому признанию эпистемической логики?

Во-первых, универсализм неразрывно связан с принципом композициональности, который говорит, что значение (или шире: семантический атрибут) сложного выражения есть функция значения (семантических атрибутов) его составных частей. Основная идея состоит в том, чтобы синтаксические правила построения формулы и семантические правила ее интерпретации работали в тандеме: предполагается, что каждому правилу, объясняющему как выражение $F$ синтаксически составлено из его составных частей, соответствует семантическое правило, говорящее как значение $F$ строится из значений составляющих его выражений. Фактически это означает выведение семантических понятий из-под власти контекста - точнее, отрицание любого другого контекста кроме того мира (в целом), который описывается, и того языка (в целом), на котором это описание осуществляется. Однако практика показывает, что принцип композициональности - довольно сильная идеализация. Возможен ли сколь-нибудь близкий к естественному язык, который был бы абсолютно контекстно-независимым? Можем ли мы игнорировать конкретность и ситуативность познания, включенность познающего субъекта в познаваемую им реальность, отрицать за ним право выражать собой определенную «перспективу видения» даже на уровне языка (об особенностях и трудностях «перспективной идентификации» см.: [5])?

Во-вторых, универсализм приводит не только к невыразимости фундаментальных семантических понятий (как показал А. Тарский, введение в язык теории предиката истинности, определяемого внутри самой этой теории, приводит к парадоксу «лжеца»), но и к невыразимости фундаментальных эпистемических понятий (еще в 1960 г. Д. Каплан и Р.Монтегю продемонстрировали, что введение в язык пропозициональной логики $K$-оператора, определяемого внутри самой этой теории, также может привести к парадоксу - т. н. «парадоксу знающего» («the knower»), разновидностью которого является «парадокс 
неожиданной казни» [10]).

В третьих, имплицитным следствием универсалистской точки зрения (тесно связанным, кстати, с принципом композициональности) является сильное синтаксическое ограничение, заложенное в самой структуре общепринятой логической нотации. А именно, области действия логических форматоров (связки, кванторы, модальные и эпистемические операторы и пр.) в привычном для нас способе записи могут находиться между собой только в антисимметричном и транзитивном отношении включения. Не допускается дисконтинуальность и взаимопересечение областей действия, что делает невозможным отображение в языке системы альтернативных форм зависимости и независимости между форматорами, которые так часто встречаются в эпистемических контекстах.

Таким образом, необходимо признать, что ситуация, помешавшая ранней эпистемической логике (т. н. эпистемической логике «первого поколения», или EL-1) завоевать достойное место в философском дискурсе, носят объективный, существенный и весьма фундаментальный характер, обусловленный парадигмальными особенностями как современной философии науки (традиция некритически противопоставлять «контексты открытия» и «контексты обоснования»), так и общепринятой символической логики (сильно идеализированное понятие рациональности, парадигма языка как «универсального посредника»).

\section{4 Эпистемическая логика «второго поколения»: задачи и трудности}

Вместе с тем, сложившееся положение дел вовсе не является фатальным - по крайней мере, со стороны самих логиков давно прикладываются усилия к тому, чтобы его преодолеть. Уже в 1960-70-х гг. были сделаны первые шаги к созданию эпистемической логики «второго поколения» (EL-2): начали интенсивно развиваться интеррогативная логика и эротетика, помимо крипкевской семантики возможных миров (PWS) появились ситуационная (SS) и теоретико-игровая семантики (GTS); в 1980-х гг. стали набирать силу идеи динамической логики и belief revision. Наконец, в 1996 г. в книге Я. Хинтикки «Пересмотренные принципы математики» впервые в систематическом виде была изложена идея так называемой IF-логики (Independence Friendly Logic), на которой мы хотели бы остановиться подробнее. Собственно, применение аппарата IF-логики и теоретико-игровой семантики 
(GTS) к традиционным проблемам логики познания Хинтикка и называет «эпистемической логикой второго поколения» (термин впервые появился в 2003 г., более развернуто концепция EL-2 излагается в его книге «Сократическая эпистемология», 2007) [8]. Схематически эволюцию идей, приведших к EL-2, можно представить так:

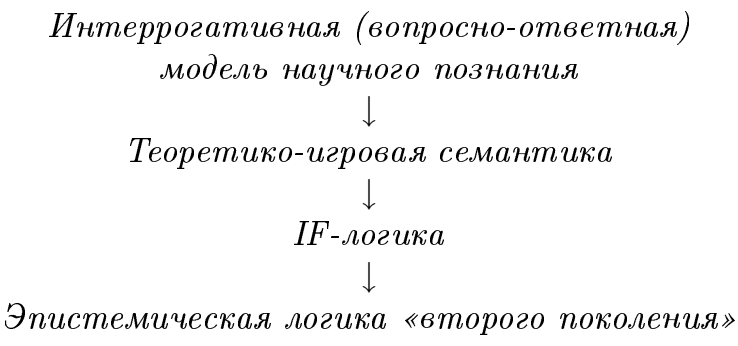

Прежде всего, EL-2 есть логика вопросов и ответов, а также поиска наилучших вопросов, которые должны быть заданы. Хинтикка резко выступает против распространенной точки зрения, утверждающей тавтологичность логических законов, и, по примеру классиков детективного жанра, пытается показать нетривиальность, информативность логических умозаключений. «Так называемые дедукции Холмca,- - утверждает он, - не сводятся к выводу эксплицитных заключений из эксплицитньх посылок. Часто он извлекает из хаоса фоновой информации нужные дополнительные посылки (сверх тех, которые, возможно, были объявлены таковыми), и уже из этих посылок по правилам самой обычной дедуктивной логики можно вывести заключения, кажущиеся на первый взгляд неожиданными» [3, с. 268].

Как же он это делает? При помощи вопросов - ведь именно вопросы служат для воплощения информации в высказывание, управляют процессом активации невербализованного знания на стадии, предшествующей собственно дедукции. Однако не следует думать, в духе платоновской теории анамнезиса, что вся фоновая информация уже заранее находится в нашей голове, и что эксплицирующие вопросы мы всегда обращаем самим себе - некоторые их посылок дедукции обнаружимы лишь в ходе наблюдений. Но большинство понятий, которые применимы к вопросам и ответам на них, применимы и к наблюдениям, так что кантовский оборот «вопросы, задаваемые природе» является не просто метафорой. «Наблюдение, - пишет Хинтикка, - это всегда ответ на вопрос» [3, с. 273]. Да и большинство дедуктивньх ходов - включая ряд наиболее интересньх - могут быть замещены соответствующим вопросом, если считать, что на него последует ответ. 
Таким образом, весь массив научного исследования - от эмпирических наблюдений до теоретических дедукций-имеет интеррогативную структуру (нет контекстов открытия и контекстов обоснования, а есть лишь различные стороны одного и того же процесса).

Вопросно-ответная последовательность как «игра с природой» становится одним из главных столпов теоретико-игровой семантики. Наличие выигрышной стратегии для Исследователя (верификатора) называется истиной, наличие выигрышной стратегии для Природы (фальсификатора) - ложью. Характерно, что в такой семантике допускается отсутствие выигрышных стратегий вообще, то есть не принимается tertium non datur (а если рассматриваются игры с ненулевой суммой, то не принимается и закон непротиворечия).

Естественно, информация о наличии выигрышной стратегии еще не является информацией о структуре этой стратегии, то есть «абстрактное» знание истинности - что-знание (th-knowledge) - вовсе не совпадает со «стратегическим» как-знанием (wh-knowledge). В частности, утверждать истинность формул с кванторами означает признавать существование соответствующих им сколемовских функций. Но от знания о наличии сколемовской функции до знания о том, как ее построить - огромная пропасть! В абстрактном фактофиксирующем дискурсе, по выражению Хинтикки, мы оперируем «долговыми расписками», которые по отношению к «живым деньгам»-стратегическим знаниям - выступают лишь как обещания [7, с. 243].

Логика стратегических аспектов истины должна быть логикой конструктивных объектов, то есть логикой идентифицирующего знания. В некотором смысле она должна быть даже более интуиционистской, чем сам традиционный интуиционизм. Общеизвестно, что интуиционистская математика была задумана для того, чтобы иметь дело не столько с математическими истинами, сколько с математическими объектами. Но просто ввести для этих целей пропозициональный $K$ оператор было явно недостаточно: ведь если само знание трактуется как исключительно пропозициональное, то вряд ли поставленная цель будет достигнута. Действительная новизна может заключаться лишь в том, чтобы от знания математических высказываний перейти к знанию математических объектов - и не потерять при этом всеобщности в трактовке знания, не позволить ему рассыпаться на два субстанциальных вида (знание de dicto и знание de re).

Как можно выразить, например, следующий факт: «а знает, кем является $b » ?$ Очевидно, вариант 
(1) $K_{a}(\exists x)(b=x)$

не подходит, т. к. в нем говорится: «a знает, что кто-то является $b$ » Гораздо корректнее вариант

(2) $(\exists x) K_{a}(b=x)$,

говорящий: «существует некто, про кого $a$ знает, что он является $b$ ». $\mathrm{C}$ точки зрения теоретико-игровой семантики формула (1) позволяет Верификатору сначала выбрать возможный мир (сценарий), узаконенный оператором $K_{a}$, а после, на базе этого выбора, приписывать значение переменной $x$. Формула (2), напротив, заставляет сначала выбрать подходящего индивида, а затем уже решать вопрос с выбором возможного сценария, совместимого со знаниями $a$.

Но ведь на самом деле, в данном случае нет никакой необходимости выбирать в рамках семантической игры $x$ до того, как выбран соответствующий $K_{a}$ сценарий! Достаточно сделать этот выбор независимо от второго выбора. Однако в синтаксисе стандартной первопорядковой логики такая возможность не предусмотрена-там все форматоры используются так, что включение одного из них в область действия другого представляет собой транзитивное и антисимметричное отношение, которое допускает ветвление только в одном направлении, и как следствие, все остальные конфигурации зависимости / независимости ассоциированных с этими форматорами переменных оказываются невыразимы.

\section{IF-логика и ее эпистемологическое значение}

Поэтому Хинтикка подчеркивает необходимость серьезного расширения выразительных возможностей классической логики, а точнее, показывает, что они долгое время были незаслуженно урезаны. В частности, это касается отношений зависимости/независимости между переменными. «Чтобы служить надежным lingua universalis, - пишет он, - ваш язык должен, во всяком случае, быть способен отобразить все возможные конфигурации зависимости и независимости между различными переменными. Ибо если некая подобная конфигурация возможна в принципе, нет никаких гарантий, что в один прекрасный день она не обнаружится среди природных, человеческих или общественных явлений, которые мы должны изучить» [6, с. 404]. 
Для этих целей им и была создана IF-логика, предметом которой являются нетрадиционные варианты информационной взаимосвязи между логическими форматорами. Самая старая и хорошо изученная разновидность конструкций подобного рода - так называемый квантор Хенкина, который обычно выражается посредством двумерной нотации:

(3) $\left|\begin{array}{l}(\forall x)(\exists y) \\ (\forall z)(\exists y)\end{array}\right\rangle F[x, y, z, u]$

Данную кванторную конструкцию невозможно выразить привычными первопорядковыми обозначениями. В теоретико-игровой интерпретации это - описание игры между двумя командами, состоящими из двух игроков. Каждый из двух членов команды Фальсификации $(F)$ выбирает по одному объекту, скажем $x$ и $z$. Затем один из членов команды Верификации $(V)$ выбирает $y$ на основании только $x$, а другой выбирает $u$, зная только $z$. Команда Верификации выигрывает если и только если объекты, выбранные таким образом, удовлетворяют $F[x, y, z, u]$. Предложение (3) истинно если и только если существует выигрышная стратегия для команды Верификации. Короче говоря, идея независимости кванторов семантически реализуется через понятие информационной независимости ходов в игре.

Конечно, предложение (3) можно записать при помощи сколемовских функций, однако для этого придется воспользоваться уже второпорядковой логикой:

(4) $(\exists f)(\exists g)(\forall x)(\forall z) F[x, f(x), z, g(z)]$

Хинтикка же, используя изобретенную им слэш-нотацию, добивается идентичного эффекта, но в первопорядковом языке (запись $\left(F_{2} y / F_{1} x\right)$ означает, что квантор $\left(F_{2} y\right)$ не зависит от квантора $\left(F_{1} x\right)$, в область действия которого он входит синтаксически):

(5) $(\forall x)(\forall z)(\exists y / \forall z)(\exists u / \forall x) F[x, y, z, u]$

По мнению Хинтикки, общепринятая первопорядковая логика, восходящая через Рассела и Уайтхеда к Фреге, не справляется со своим предназначением. «Пожалуй, ее стоило бы назвать „ограниченно-независимостной“ или, более точно, „нетерпимой к независимости“ первопорядковой логикой», - пишет он [6, с. 408]. Что же касается IF-логики, то ее правильнее называть «гиперклассической». Специфицирующий эпитет «дружественная к независимости» на самом деле не 
очень подходит для нее, так как IF-логика дружественна не только к независимости, но и к зависимости - в том плане, что она позволяет выразить кванторные конструкции, содержащие больше зависимостей, чем это можно выразить в обычной логике. Последнюю, стало быть, можно также назвать «ограниченно-зависимостной».

Хорошей иллюстрацией может служить случай с двумя взаимозависимыми кванторами, как, например, в формуле

(6) $(\forall t)(\forall x)(\forall y)(\exists z / \forall x)(\exists u / \forall y)((x=z) \wedge(y=u) \wedge F[t, x, y])$

Если представить, что $F[t, x, y]$ - некое уравнение, описывающее определенные физические феномены, то данная формула утверждает, что значения $x$ и $y$ в некий определенный момент $t$ не могут быть предсказаны независимо друг от друга. Иными словами, IF-логика показывает, как можно логическими средствами работать с феноменом неопределенности, известным из квантовой механики.

Возвращаясь к вопросу о том, как возможна логика wh-знания, мы можем теперь вполне адекватным образом выразить факт «a знает, кем является $b »$, при помощи формулы:

(7) $K_{a}\left(\exists x / K_{a}\right)(b=x)$

Соответственно, факт «a знает, какую именно функцию представляет собой $f$ », записывается в виде:

(8) $(\exists g) K_{a}(f=g)$, что является сокращением для

(9) $(\exists g) K_{a}(\forall)(f(x)=g(x))$,

и в свою очередь, эквивалентно первопорядковой формуле

(10) $K_{a}(\forall)\left(\exists / K_{a}\right)(f(x)=y)$

Таким образом, использование IF-логики и связанной с ней теоретико-игровой семантики позволяет добиться сразу трех целей, без которых проект EL-2 не мог бы быть завершен:

1) Включить познающего субъекта в область действия $K$-оператора (представить ситуативное, перспективалистское wh-знание как результат активных действий субъекта - его ходов в семантических играх с несовершенной информацией), 
2) Редуцировать wh-знание к th-знанию (репрезентировать их с помощью одного и того же пропозиционального оператора $K$ ),

3) Выразить полученные эпистемические структуры на первопорядковом языке, избежав онтологических обязательств, навязываемых логикой второго порядка (ведь очевидно, что адекватная эпистемическая логика должна быть онтологически нейтральной).

\section{6 Вызов результатам Тарского}

Интересной, но вполне предсказуемой особенностью IF-логики является то, что ее язык позволяет сформулировать предикат истинности для самой этой теории, поколебав тем самым общепринятую трактовку знаменитой теоремы Тарского о невыразимости истины (см.: $[2,7])$. Все, что для этого необходимо - чтобы язык теории был способен описать собственный синтаксис. Поскольку истинность формул с кванторами заключается в существовании соответствующих сколемовских функций, а суждения об этом, благодаря способности IF-логики выражать все возможные структуры зависимости/независимости между кванторами, переводимы на ее собственный язык (как, например, (8) и (10)), мы легко можем в самом этом языке формулировать Тэквивалентности Тарского.

Разумеется, под «определением истинности» здесь имеется в виду лишь фактофиксирующая сторона данного понятия, но отнюдь не стратегическая. Ведь знать, что некое высказывание истинно, и знать, каким образом оно делается истинным - не одно и то же.

Впрочем, действительно ли в IF логике удается «снять заклятие Тарского» и устранить проблему иерархии языков? Пусть IF язык содержит свое собственное определение истинности, причем это определение является адекватным в смысле Тарского. Не возникает ли сходная «иерархическая» проблема с самим понятием адекватности? В работе [11] приводится доказательство теоремы о том, что если L фундаментальный IF язык, то понятия логической истинности, логической импликации и логической эквиваленции для $\mathrm{L}$ неопределимы какой бы то ни было формулой конечного порядка, имеющей ту же самую сигнатуру, что и L. Иными словами, даже если понятие истинности для языка L, стандартным образом (по Тарскому) сконструированное в метаязыке M, адекватно переводимо обратно в язык L, как утверждает Хинтикка,- что с того? «Мы, носители языка М, будем 
знать [что оно действительно адекватно], но они [носители языка L] не смогут этого знать» [11].

Заслуживает также отдельного внимания вопрос о том, насколько первопорядковая IF-логика действительно может называться первопорядковой. Как замечает финский логик, «шаг от первопорядковой логики обычного типа к IF-логике не меняет решительно ничего, и уж определенно он не меняет классы значений, по которым пробегают наши переменные» [6, с. 410]. Однако вряд ли все так просто. Ведь если в IF-языке действительно выразимы Т-эквивалентности Тарского, значит одно и то же суждение, использующее индивидную переменную $x$, можно трактовать и как утверждение об объекте $x$ и как утверждение о функции выбора, соответствующей этому объекту. Сам Хинтикка в оправдание объектной интерпретации ссылается на критерий Куайна, однако вопрос о том, насколько применим этот критерий в данном случае, и применим ли вообще, остается открытым. Следует признать, что само понятие «первопорядковой логики» нуждается сегодня в серьезном пересмотре.

Подводя итог, хотелось бы отметить, что

1. Эпистемическая логика «второго поколения» успешно преодолела все недостатки систем «первого поколения» и является на сегодняшний день весьма тонким и эффективным механизмом анализа человеческого познания.

2. Это стало возможным лишь благодаря исследованию до-теоретических предпосылок, лежащих в основе классической логики как таковой (в том числе, предпосылок эпистемологического характера) и их деконструкции.

3. Движение от логики эпистемологии к эпистемологии логики заставляет переосмыслить классические теоремы об ограниченности выразительных и дедуктивных возможностей формальных теорий - по крайней мере, дать им новую философскую интерпретацию.

\section{7 Литература}

[1] Поппер К. Логика и рост научного знания. - М., 1983.

[2] Хинтикка Я. Проблема истины в современной философии // Вопросы философии. - 1996. - № 9 . 
[3] Хинтикка Я., Хинтикка М. Шерлок Холмс против современной логики: к теории поиска информации с помощью вопросов. // Язык и моделирование социального взаимодействия. Переводы. Сост. В.М. Сергеева и П.Б. Паршина. Общ. ред. В.В. Петрова. М.: Прогресс, 1987.

[4] Heijenoort van J., Logic as calculus and logic as language // Synthese. - 17. - 1967.

[5] Hintikka J. Perspectival identification, demonstratives and "small worlds" // Synthese. - 114. - 1998.

[6] Hintikka J. Hyperclassical logic (a.k.a. IF Logic) and it's implications for logical theory. // The Bulletin of Symbolic Logic. - Vol.8. - № 3. 2002.

[7] Hintikka J. What is truth? Stay for an answer // Current Issues in Theoretical Philosophy: What is Truth? (ed. R. Schantz). - Walter de Gruyter, 2002.

[8] Hintikka J. A second-generation epistemic logic and its general significance // Hintikka J., Socratic Epistemology: Explorations of Knowledge-Seeking by Questioning. - Cambridge University Press, 2007.

[9] Hocutt M. Is epistemic logic possible? // Notre Dame Journal of Formal Logic. - 13. - 1972.

[10] Kaplan D., Montague R. A paradox regained // Notre Dame Journal of Formal Logic. - Vol.1. - № 3. - 1960.

[11] Rouilhan P., Bozon S. The truth of IF: Has Hintikka really exorcised Tarski's curse? // The Philosophy of Jaakko Hintikka. - Open Court, 2006. 